\title{
Bruxism- is botulinum toxin an effective treatment?
}

\author{
Abstracted from \\ De la Torre Canales G, Câmara-Souza MB, do Amaral CF, Garcia RC, Manfredini D.
}

Is there enough evidence to use botulinum toxin injections for bruxism management? A systematic literature review. Clin Oral Investig 2017; 21: 727-734. doi: 10.1007/s00784-017-2092-4. Review. PubMed PMID: 28255752.

Address for correspondence: Renata Cunha Matheus Rodrigues Garcia, Department of Prosthodontics and Periodontology, Piracicaba Dental School, University of Campinas, Avenida Limeira, no. 901, Bairro Areiafio, Piracicaba, SP 13414-903, Brazil. E-mail: regarcia@fop.unicamp.br

\section{Question: Is botulinum toxin an option for the treatment of bruxism?}

Data sources PubMed, Scopus, Web of Science, Embase, Cochrane, Scielo and Lilacs databases were searched from 1980 to March 2016. Literature reviews were explored in order to retrieve any other relevant papers.

Study selection Randomised controlled studies (RCTs), prospective and before and after studies that applied botulinum toxin (BoNT-A) to the masseter and/or temporalis muscles assessing objective and subjective parameters of bruxism published in English were included. Data extraction and synthesis Three reviewers independently assessed for eligibility. Disagreement was solved by discussion and when reaching consensus between at least two. Standard data items were extracted. Quality assessment was assessed using the Cochrane Collaboration's risk of bias tool and the Critical Appraisal Skills Program (CASP) tool. Before-After Study Checklist was used for the nonrandomised studies. Results were presented as a narrative review. Results Five studies were included in the review, of those three were RCTs and two before and after. A total of 188 subjects were included. Regimen was unique in each study and the follow-up ranged from two to 24 weeks. The risk of bias of the RCTs was assessed as low to unclear, while the before and after studies were assessed as of moderate risk of bias.

Conclusions The authors concluded that botulinum toxin represents a possible option for the management of sleep bruxism (SB), minimising symptoms and reducing the intensity of contractions rather than for SB itself.

\section{Commentary}

The review intended to summarise the effect of botulinum toxin injections in the management of bruxism. BoNT-A is emerging as a possible treatment modality for bruxism based on the potential effect on muscle activity. The limited number of studies identified may be due in part to the fact that it is a relatively novel therapeutic approach.

The authors performed a comprehensive search for only English papers that included two non-English databases.

One fundamental issue with the topic is the lack of consensus about the definition of bruxism. The latest attempt came in 2013 with the publication of what the authors called 'an international consensus' paper that defined bruxism as 'a repetitive jaw-muscle activity characterised by clenching or grinding of the teeth and/or by bracing or thrusting of the mandible. Bruxism has two distinct circadian manifestations: it can occur during sleep (indicated as sleep bruxism) or during wakefulness (indicated as awake bruxism). ${ }^{1}$ Although this definition is still pending validation, it is important to highlight it does not include any subjective symptom and does not refer to the chronic nature of the condition.

Polysomnography (PSG) is considered the gold standard for SB diagnosis and was used in a single study in the review $(n=20)$. Because the test is expensive and time-consuming, the majority of studies on the topic rely on self-reporting bruxism instead. On the use of self-report Raphael in 2015 found that self-report of tooth grinding awareness is highly unlikely to be a valid indicator of true $\mathrm{SB} .^{2}$ This controversy will require further elucidation.

Another fundamental issue requiring clarification is the relationship between the intensity of muscle contractions and pain. It is unclear if pain is a valid surrogate to assess the effect of a treatment on muscle activity.

Other limitations on the present evidence are based on: variations on the diagnostic criteria for each of the included studies; the size of the population being small in four out of five studies with unclear power to detect a difference; each study following a unique therapeutic protocol; the follow up varying from two to 24 weeks and the outcome being different for each study.

From the five included studies three were RCTs, of those two were placebo controlled $(\mathrm{n}=32)$ while one compared two interventions $(\mathrm{n}=20)$ : injection into the masseter versus injection into the masseter and the temporalis. The risk of bias was assessed as unclear for the majority of items from each study. The quality score of the two before and after studies $(n=132)$ was assessed as moderate. It is unclear which CASP tool was used.

The present evidence points to possible benefits on self-reported outcomes but the quality of the evidence is low and there are some fundamental issues requiring clarification. Other issues that need to be considered are cost, side effects and long term effects.

Arthi Kumar and Silvia Spivakovsky New York University College of Dentistry, New York, USA

1. Lobbezoo F, Ahlberg J, Glaros AG, et al. Bruxism defined and graded: an international consensus. J Oral Rehabil 2013; 40: 2-4.

2. Raphael KG, Janal MN, Sirois DA, et al. Validity of self-reported sleep bruxism among myofascial temporomandibular disorder patients and controls. J Oral Rehabil 2015; 42: $751-758$. doi:10.1111/joor.12310.

Evidence-Based Dentistry (2018) 19, 59. doi:10.1038/sj.ebd.6401311 